\title{
NON-EXISTENCE OF EXCHANGE TRANSFORMATIONS OF ITERATED JET FUNCTORS
}

\author{
MIROSLAV DOUPOVEC \\ Department of Mathematics, Brno University of Technology \\ FSI VUT Brno, Technická 2, 61669 Brno, Czech Republic \\ E-mail:doupovec@fme.vutbr.cz \\ WŁODZIMIERZ M. MIKULSKI \\ Institute of Mathematics, Jagiellonian University \\ Reymonta 4, 30-059 Kraków, Poland \\ E-mail:mikulski@im.uj.edu.pl
}

\begin{abstract}
We study the problem of the non-existence of natural transformations $J^{r} J^{s} Y \rightarrow$ $J^{s} J^{r} Y$ of iterated jet functors depending on some geometric object on the base of $Y$.
\end{abstract}

1. Introduction. It is well known that for every couple $F$ and $G$ of product preserving bundle functors defined on the category $\mathcal{M f}$ of smooth manifolds and all smooth maps there is an exchange natural equivalence $\kappa^{F, G}: F G \rightarrow G F$, [5]. Moreover, denoting by $p_{M}^{F}: F M \rightarrow M$ and $p_{M}^{G}: G M \rightarrow M$ the bundle projections, we have $p_{F M}^{G} \circ \kappa_{M}^{F, G}=F\left(p_{M}^{G}\right)$, i.e. $\kappa_{M}^{F, G}$ interchanges the projections $p_{F M}^{G}$ and $F\left(p_{M}^{G}\right)$. We remark that this property generalizes the classical involution $\kappa_{M}^{T, T}: T T M \rightarrow T T M$ of the iterated tangent bundle to every pair $F$ and $G$ of product preserving functors on $\mathcal{M f}$.

In $[2]$ we applied this point of view to natural equivalences

$$
A^{F, G}: F G \rightarrow G F
$$

of iterated bundle functors defined on the category $\mathcal{F} \mathcal{M}_{m}$ of fibered manifolds with $m$-dimensional bases and of fibered manifold morphisms covering local diffeomorphisms. In particular, we have extended the concept of the canonical involution in the following way. Given an arbitrary fibered manifold $Y \rightarrow M$, we denote by $p_{Y}^{F}: F Y \rightarrow Y$ and $p_{Y}^{G}: G Y \rightarrow Y$ the bundle projections. Then the natural equivalence $A^{F, G}$ is called the

2000 Mathematics Subject Classification: 58A05, 58A20.

Key words and phrases: natural transformation, jet prolongation, symplectic structure.

The first author was supported by a grant of the GA ČR No 201/05/0523.

The paper is in final form and no version of it will be published elsewhere. 
involution, if $p_{F Y}^{G} \circ A_{Y}^{F, G}=F\left(p_{Y}^{G}\right)$ for an arbitrary fibered manifold $Y \rightarrow M$. By [2], [6] and [9], involutions can be applied in the prolongation of connections.

An important example of a bundle functor on $\mathcal{F} \mathcal{M}_{m}$ is the $r$-th jet functor $J^{r}$, which associates to an arbitrary fibered manifold $Y \rightarrow M$ its $r$-th jet prolongation $J^{r} Y \rightarrow M$. For such functors we have proved that for any $r$ and $s$ there is no involution $J^{r} J^{s} \rightarrow J^{s} J^{r}$, [2]. On the other hand, M. Modugno [9] has introduced the involution $\operatorname{ex}_{\Lambda}: J^{1} J^{1} \rightarrow J^{1} J^{1}$ depending on a classical linear connection $\Lambda$ on the base manifold $M$.

In this paper we study the more general problem on the non-existence of natural transformations (not necessarily involutions)

$$
\left(A_{\sigma}\right)_{Y}: J^{r} J^{s} Y \rightarrow J^{s} J^{r} Y
$$

depending on some geometric object $\sigma$ on the base of $Y$. The main result will be proved in Section 1. Further, in Section 2 we prove that for $r \neq s$ there is no natural transformation $\left(A_{\omega}\right)_{Y}: J^{r} J^{s} Y \rightarrow J^{s} J^{r} Y$ depending on a symplectic form $\omega$ on the base of $Y$. As a direct consequence we obtain that for $r \neq s$ there is no natural transformation $J^{r} J^{s} Y \rightarrow J^{s} J^{r} Y$. Finally, Section 3 is devoted to the problem of the non-existence of non-trivial natural transformations $\left(A_{\omega}\right)_{Y}: J^{r} J^{r} Y \rightarrow J^{r} J^{r} Y$.

We remark that higher order jet functors play an important role in differential geometry, see e.g. [3], [4] and [7]. In what follows we use the terminology and notation from the book [5]. We denote $\mathcal{M} f_{m} \subset \mathcal{M} f$ the subcategory of $m$-dimensional manifolds and their local diffeomorphisms and by $\mathcal{F} \mathcal{M}_{m, n} \subset \mathcal{F} \mathcal{M}_{m}$ the subcategory of fibered manifolds with $n$-dimensional fibres and their local fibered diffeomorphisms. All manifolds and maps are assumed to be infinitely differentiable.

2. The main result. Let $F$ be a natural bundle on $\mathcal{M} f_{m}$. Given a manifold $M$ we denote by $\Gamma_{\text {loc }}(F M)$ the set of local smooth sections of $F M$. Further, suppose that for all $\mathcal{M} f_{m}$-objects $M$ we have $D(M) \subset \Gamma_{\text {loc }}(F M)$ such that from $\sigma \in D\left(M_{1}\right)$ it follows that $F \psi \circ \sigma \circ \psi^{-1} \in D\left(M_{2}\right)$ for any $\mathcal{M} f_{m}$-map $\psi: M_{1} \rightarrow M_{2}$.

Definition 1. An $\mathcal{F} \mathcal{M}_{m, n}$-natural operator $A: D \rightsquigarrow\left(J^{r} J^{s}, J^{s} J^{r}\right)$ is an invariant family of functions

$$
A: D(M) \rightarrow C_{M}^{\infty}\left(J^{r} J^{s} Y, J^{s} J^{r} Y\right)
$$

into the space $C_{M}^{\infty}\left(J^{r} J^{s} Y, J^{s} J^{r} Y\right)$ of all base preserving maps $J^{r} J^{s} Y \rightarrow J^{s} J^{r} Y$ for any $\mathcal{F} \mathcal{M}_{m, n}$-object $Y \rightarrow M$. The invariance means that for any $\mathcal{F} \mathcal{M}_{m, n}$-objects $Y_{1} \rightarrow M_{1}$ and $Y_{2} \rightarrow M_{2}$ and any $\mathcal{F} \mathcal{M}_{m, n}$-map $\Psi: Y_{1} \rightarrow Y_{2}$ covering $\psi: M_{1} \rightarrow M_{2}$ and any sections $\sigma_{1} \in D\left(M_{1}\right)$ and $\sigma_{2} \in D\left(M_{2}\right)$ from $\sigma_{2} \circ \psi=F \psi \circ \sigma_{1}$ it follows that $A\left(\sigma_{2}\right) \circ J^{r} J^{s} \Psi=$ $J^{s} J^{r} \Psi \circ A\left(\sigma_{1}\right)$.

In the special case $D(M)=\Gamma_{\text {loc }}(F M)$ we write $A: F \rightsquigarrow\left(J^{r} J^{s}, J^{s} J^{r}\right)$ instead of $A: D \rightsquigarrow\left(J^{r} J^{s}, J^{s} J^{r}\right)$. The main result of the present paper is the following non-existence theorem.

THEOREM 1. Let $r$ and $s$ be natural numbers such that $r>s$. Let $F$ and $D$ be as above. Suppose that there exists a section $\rho \in D\left(\mathbf{R}^{m}\right)$ and an $\mathcal{M} f_{m}-$ map $\varphi=\left(\varphi^{i}\right): \mathbf{R}^{m} \rightarrow \mathbf{R}^{m}$ such that 
(a) $F \varphi \circ \rho \circ \varphi^{-1}=\rho$ near $0 \in \mathbf{R}^{m}$,

(b) $j_{0}^{r+s-1} \varphi=i d$,

(c) $\varphi^{1}$ depends only on $x^{1}$ and

(d) $\frac{d^{r+s}}{d\left(x^{1}\right)^{r+s}} \varphi^{1}(0) \neq 0$.

Then there is no $\mathcal{F} \mathcal{M}_{m, n}$-natural operator $A: D \rightsquigarrow\left(J^{r} J^{s}, J^{s} J^{r}\right)$.

Proof. Denoting by $\mathbf{R}^{m, n}$ the product fibered manifold $\mathbf{R}^{m} \times \mathbf{R}^{n} \rightarrow \mathbf{R}^{m}$, we identify sections of $\mathbf{R}^{m, n}$ with maps $\mathbf{R}^{m} \rightarrow \mathbf{R}^{n}$. Further, we use the notation

$$
j_{0}^{r} j^{s}(f(x, \underline{x}))=j_{0}^{r}\left(x \rightarrow j_{x}^{s}(\underline{x} \rightarrow f(x, \underline{x}))\right) \in J_{0}^{r} J^{s} \mathbf{R}^{m, n} .
$$

Suppose that there exists an operator $A$ in question. Consider first the restriction $\tilde{A}$ : $J_{0}^{r} J^{s} \mathbf{R}^{m, n} \rightarrow J_{0}^{s} J^{r} \mathbf{R}^{m, n}$ of $A(\rho)$ to the fibers over $0 \in \mathbf{R}^{m}$. Using the invariance of $\tilde{A}$ with respect to the fiber homotheties of $\mathbf{R}^{m, n}$ and the homogeneous function theorem from [5] we see that $\tilde{A}$ is linear. Taking into account the invariance of $\tilde{A}$ with respect to the $\mathcal{F} \mathcal{M}_{m, n}$-maps

$$
\left(x^{1}, \ldots, x^{m}, y^{1}, t y^{2}, \ldots, t y^{n}\right)
$$

for $t \neq 0$ we can write

$$
\tilde{A}\left(j_{0}^{r} j^{s}\left(x^{1}, 0, \ldots, 0\right)\right)=j_{0}^{s} j^{r}\left(\sum_{|\alpha| \leq s} \sum_{|\beta| \leq r} a_{\alpha \beta} x^{\alpha}(\underline{x}-x)^{\beta}, 0, \ldots, 0\right)
$$

for some uniquely determined $a_{\alpha \beta} \in \mathbf{R}$ for $m$-tuples $\alpha, \beta$ with $|\alpha| \leq s,|\beta| \leq r$. Further, considering the invariance of $\tilde{A}$ with respect to the $\mathcal{F} \mathcal{M}_{m, n}$-map

$$
\left(x^{1}, \ldots, x^{m}, y^{1}-x^{1}, y^{2}, \ldots, y^{n}\right)
$$

we get from (1)

$$
\tilde{A}\left(j_{0}^{r} j^{s}\left(x^{1}-\underline{x}^{1}, 0, \ldots, 0\right)\right)=j_{0}^{s} j^{r}\left(\sum_{|\alpha| \leq s} \sum_{|\beta| \leq r} a_{\alpha \beta} x^{\alpha}(\underline{x}-x)^{\beta}-\underline{x}^{1}, 0, \ldots, 0\right) .
$$

Then the invariance of $\tilde{A}$ with respect to the $\mathcal{F} \mathcal{M}_{m, n}$-map

$$
\left(x^{1}, \ldots, x^{m}, y^{1}+\left(y^{1}\right)^{s+1}, y^{2}, \ldots, y^{n}\right)
$$

and the linearity of $\tilde{A}$ yield

$$
\begin{aligned}
\tilde{A}\left(j_{0}^{r} j^{s}\left(\left(x^{1}-\underline{x}^{1}\right)^{s+1}, 0, \ldots, 0\right)\right) & \\
& =j_{0}^{s} j^{r}\left(\left(\sum_{|\alpha| \leq s} \sum_{|\beta| \leq r} a_{\alpha \beta} x^{\alpha}(\underline{x}-x)^{\beta}-\underline{x}^{1}\right)^{s+1}, 0, \ldots, 0\right) .
\end{aligned}
$$

But $j_{0}^{r} j^{s}\left(\underline{x}^{1}-x^{1}\right)^{s+1}=0$ as $j_{x}^{s}\left(\underline{x}^{1}-x^{1}\right)^{s+1}=0$. Then from (2) we get

$$
j_{0}^{s} j^{r}\left(\sum_{|\alpha| \leq s} \sum_{|\beta| \leq r} a_{\alpha \beta} x^{\alpha}(\underline{x}-x)^{\beta}-\underline{x}^{1}\right)^{s+1}=0,
$$

which reads $a_{(0)(0)}=0$ and

$$
j_{0}^{s} j^{r}\left(\left(a_{(0) e_{1}}-1\right)^{s+1}\left(\underline{x}^{1}-x^{1}\right)^{s+1}+\sum_{l=1}^{m}\left(a_{(0) e_{l}}\right)^{s+1}\left(\underline{x}^{l}-x^{l}\right)^{s+1}+\ldots\right)=0,
$$


where the dots denote the linear combination of terms $x^{\gamma}(\underline{x}-x)^{\eta}$ for other $(\gamma, \eta),|\gamma| \leq s$, $|\eta| \leq r$. Since $r \geq s+1$ (because of the assumption $r>s$ ), we have

$$
a_{(0) e_{1}}=1 \text { and } a_{(0) e_{l}}=0 \text { for } l=2, \ldots, m \text {, }
$$

where $e_{j}=(0, \ldots, 1, \ldots, 0)$ is the $m$-tuple with 1 in the $j$-th position. Then (by (4) and the assumptions (b) and (c)), the map $\varphi^{-1} \times \operatorname{id}_{\mathbf{R}^{n}}$ sends

$$
j_{0}^{s} j^{r}\left(\sum_{|\alpha| \leq s} \sum_{|\beta| \leq r} a_{\alpha \beta} x^{\alpha}(\underline{x}-x)^{\beta}\right)
$$

into

$$
j_{0}^{s} j^{r}\left(\sum_{|\alpha| \leq s} \sum_{|\beta| \leq r} a_{\alpha \beta} x^{\alpha}(\underline{x}-x)^{\beta}+\frac{1}{(r+s) !} \frac{d^{r+s}}{d\left(x^{1}\right)^{r+s}} \varphi^{1}(0)\left(\underline{x}^{1}\right)^{r+s}+\ldots\right),
$$

where the dots denote an expansion of terms $\left(x^{1}\right)^{r+s}$ and $x^{\gamma} \underline{x}^{\eta}$ with $|\gamma+\eta|>r+s$. Of course,

$$
j_{0}^{s} j^{r}\left(\underline{x}^{1}\right)^{r+s} \neq 0 \text { and } j_{0}^{s} j^{r}\left(x^{1}\right)^{r+s}=0 .
$$

By the Newton formula, $j_{0}^{s} j^{r}\left(x^{\gamma} \underline{x}^{\eta}\right)=j_{0}^{s} j^{r}\left(x^{\gamma}(\underline{x}-x+x)^{\eta}\right)$ is the linear combination of terms $j_{0}^{s} j^{r}\left(x \underline{\gamma}(\underline{x}-x)^{\underline{\eta}}\right)$ with $|\underline{\gamma}+\eta|=|\gamma+\eta|$ (which are zero if $|\gamma+\eta|>r+s$ ). Then the dots in (5) are zero. Therefore $\varphi^{-1} \times \operatorname{id}_{\mathbf{R}^{n}}$ does not preserve the right hand side of (1) because $\frac{d^{r+s}}{d\left(x^{1}\right)^{r+s}} \varphi^{1}(0) \neq 0$ (assumption $\left.(\mathrm{d})\right)$. On the other hand $\varphi^{-1} \times i d_{\mathbf{R}^{n}}$ preserves the left hand side of (1) because it preserves both the section $\rho$ (assumption (a)) and $j_{0}^{r} j^{s}\left(x^{1}, 0, \ldots, 0\right)$ (as $j_{0}^{r} \varphi=$ id because of assumption (b)). This is a contradiction.

Corollary 1. Let $F$ be a natural vector bundle. Then there is no $\mathcal{F M}_{m, n}$-natural operator $A: F \rightsquigarrow\left(J^{r} J^{s}, J^{s} J^{r}\right)$ for $r>s$.

Proof. It follows from Theorem 1 with $\rho=0$.

Corollary 2. For $r>s$ there is no $\mathcal{F} \mathcal{M}_{m, n}$-natural transformation $B: J^{r} J^{s} \rightarrow J^{s} J^{r}$.

Proof. Any such $B: J^{r} J^{s} \rightarrow J^{s} J^{r}$ can be treated as the corresponding constant $\mathcal{F} \mathcal{M}_{m, n^{-}}$ natural operator $B: T \rightsquigarrow\left(J^{r} J^{s}, J^{s} J^{r}\right)$.

Open problem. Clearly, for $r=s$ Theorem 1 does not hold (we have the identity map $\left.J^{r} J^{r} Y \rightarrow J^{r} J^{r} Y\right)$. On the other hand, we do not know whether Theorem 1 is true in the case $r<s$.

\section{Natural transformations $J^{r} J^{s} \rightarrow J^{s} J^{r}$ depending on a symplectic structure.} From Theorem 1 we obtain easily

Proposition 1. For $r>s$ there is no $\mathcal{F M}_{2 \underline{m}, n}$-natural operator $A: S Y M P \rightsquigarrow\left(J^{r} J^{s}, J^{s} J^{r}\right)$ transforming symplectic structures $\omega$ on $M$ into natural transformations $A(\omega): J^{r} J^{s} Y \rightarrow$ $J^{s} J^{r} Y$. 
Proof. In Theorem 1 we put $m=2 \underline{m}, F=\bigwedge^{2} T^{*}$,

$D(M)=\operatorname{SYMP}(M)=$ the space of local symplectic structures on $M$,

$$
\begin{aligned}
\rho & =\sum_{i=1}^{\underline{m}} d x^{i} \wedge d x^{\underline{m}}+i=\text { the standard symplectic structure on } \mathbf{R}^{2 \underline{m}}, \\
\varphi & =\left(x^{1}+\frac{1}{r+s}\left(x^{1}\right)^{r+s}, x^{2}, \ldots, x^{\underline{m}}, \frac{x^{\underline{m}}+1}{1+\left(x^{1}\right)^{r+s-1}}, x^{\underline{m}+2}, \ldots, x^{2 \underline{m}}\right) .
\end{aligned}
$$

We can see that $\varphi$ and $\rho$ satisfy assumptions (a)-(d) of Theorem 1 .

Now we prove

Proposition 2. For $s>r$ there is no $\mathcal{F} \mathcal{M}_{2 \underline{m}, n}$-natural operator $A: S Y M P \rightsquigarrow\left(J^{r} J^{s}, J^{s} J^{r}\right)$ transforming symplectic structures $\omega$ on $M$ into natural transformations $A(\omega): J^{r} J^{s} Y \rightarrow$ $J^{s} J^{r} Y$.

Proof. Suppose that there exists $A$ in question. Let

$$
\tilde{A}: J_{0}^{r} J^{s} \mathbf{R}^{2 \underline{m}, n} \rightarrow J_{0}^{s} J^{r} \mathbf{R}^{2 \underline{m}, n}
$$

be the restriction of $A\left(\omega^{o}\right)$ to the fiber over $0 \in \mathbf{R}^{2} \underline{m}$, where $\omega^{o}=\sum_{\tilde{\alpha}} \frac{m}{i=1} d x^{i} \wedge d x^{\underline{m}}+i$ is the standard symplectic structure on $\mathbf{R}^{2 \underline{m}}$. Using the invariance of $\tilde{A}$ with respect to the fiber homotheties of $\mathbf{R}^{2 \underline{m}, n}$ and the homogeneous function theorem we see that $\tilde{A}$ is linear. Next, considering the invariance of $\tilde{A}$ with respect to the $\mathcal{F} \mathcal{M}_{2 \underline{m}, n}$-maps

$$
\left(\tau_{1} x^{1}, \ldots, \tau_{\underline{m}} x^{\underline{m}}, \frac{1}{\tau_{1}} x^{\underline{m}+1}, \ldots, \frac{1}{\tau_{\underline{m}}} x^{2 \underline{m}}, y^{1}, \tau y^{2}, \ldots, \tau y^{n}\right)
$$

preserving $\omega^{o}$ one can easily show that

$$
\tilde{A}\left(j_{0}^{r} j^{s}\left(x^{1}, 0, \ldots, 0\right)\right)=j_{0}^{s} j^{r}\left(a x^{1}+b \underline{x}^{1}+\ldots, 0, \ldots, 0\right),
$$

where the dots mean some combination of monomials in $x, \underline{x}$ of degree $\geq 2$. Taking into account the invariance of $\tilde{A}$ with respect to the $\mathcal{F} \mathcal{M}_{2 \underline{m}, n}$-map

$$
\left(x^{1}+\left(x^{1}\right)^{r+1}, x^{2}, \ldots, x^{\underline{m}}, \frac{x^{\underline{m}+1}}{1+(r+1)\left(x^{1}\right)^{r}}, x^{\underline{m}+2}, \ldots, x^{2 \underline{m}}, y^{1}, \ldots, y^{n}\right)^{-1}
$$

preserving $\omega^{o}$ and $j_{0}^{r} j^{s} x^{1}$ we deduce

$$
j_{0}^{s} j^{r}\left(a\left(x^{1}\right)^{r+1}+b\left(\underline{x}^{1}\right)^{r+1}+\ldots, 0, \ldots, 0\right)=0,
$$

where the dots denote some expression of monomials of degree $\geq r+2$. Applying $\left(t \operatorname{id}_{\mathbf{R}^{2} \underline{m}} \times\right.$ $\left.\operatorname{id}_{\mathbf{R}^{n}}\right)$ to both sides of (6) we get

$$
j_{0}^{s} j^{r}\left(a\left(x^{1}\right)^{r+1}+b\left(\underline{x}^{1}\right)^{r+1}, 0, \ldots, 0\right)=0 .
$$

But

$$
j_{0}^{s} j^{r}\left(\underline{x}^{1}\right)^{r+1}=j_{0}^{s} j^{r}\left(\underline{x}^{1}-x^{1}+x^{1}\right)^{r+1}=j_{0}^{s} j^{r}\left(\sum_{k=0}^{r} C_{k}^{r+1}\left(\underline{x}^{1}-x^{1}\right)^{k}\left(x^{1}\right)^{r+1-k}\right) .
$$

From (7) and the assumption $s>r$ we get $a+b=0$ and $b=0$. Then we have

$$
\tilde{A}\left(j_{0}^{r} j^{s}\left(x^{1}, 0, \ldots, 0\right)\right)=j_{0}^{s} j^{r}(*, 0, \ldots, 0),
$$


where $*$ denote some linear combination of monomials in $x, \underline{x}$ of degree $\geq 2$. Further, using the invariance of $\tilde{A}$ with respect to the $\mathcal{F} \mathcal{M}_{2 \underline{m}, n}$-map

$$
\left(x^{1}, \ldots, x^{2 \underline{m}}, y^{1}-x^{1}, y^{2}, \ldots, y^{n}\right)
$$

preserving $\omega^{o}$ we get from (8)

$$
\tilde{A}\left(j_{0}^{r} j^{s}\left(x^{1}-\underline{x}^{1}, 0, \ldots, 0\right)\right)=j_{0}^{s} j^{r}\left(-\underline{x}^{1}+*, 0, \ldots, 0\right) .
$$

Then using the invariance of $\tilde{A}$ with respect to the $\mathcal{F} \mathcal{M}_{2 \underline{m}, n}$-map

$$
\left(x^{1}, \ldots, x^{2 \underline{m}}, y^{1}+\left(y^{1}\right)^{s+1}, y^{2}, \ldots, y^{n}\right)
$$

we obtain from $(9)$

$$
\tilde{A}\left(j_{0}^{r} j^{s}\left(\left(\underline{x}^{1}-x^{1}\right)^{s+1}, 0, \ldots, 0\right)\right)=j_{0}^{s} j^{r}\left(\left(\underline{x}^{1}\right)^{s+1}+* *, 0, \ldots, 0\right),
$$

where $^{* *}$ is some linear combination of monomials in $x, \underline{x}$ of degree $\geq s+2$. But $j_{0}^{r} j^{s}\left(\left(\underline{x}^{1}-\right.\right.$ $\left.\left.x^{1}\right)^{s+1}\right)=0$. So from (10) we have

$$
j_{0}^{s} j^{r}\left(\left(\underline{x}^{1}\right)^{s+1}\right)=0 .
$$

This is a contradiction as $j_{0}^{s} j^{r}\left(\left(\underline{x}^{1}\right)^{s+1}\right)=j_{0}^{r+s}\left(\left(\underline{x}^{1}\right)^{s+1}\right) \neq 0$.

From Corollary 2 and Proposition 3 we obtain

Proposition 3. For $r \neq s$ there is no natural transformation $A: J^{r} J^{s} \rightarrow J^{s} J^{r}$.

Proof. It suffices to prove the case $s>r$. Obviously, such $A$ can be treated as a natural operator $A$ : SYMP $\rightsquigarrow\left(J^{r} J^{s}, J^{s} J^{r}\right)$ constant with respect to elements from SYMP. By Proposition 3 the proof is complete.

It is interesting to point out that the only natural transformation $J^{r} J^{s} \rightarrow J^{r} J^{s}$ is the identity, [1].

4. Non-identical natural transformations $J^{r} J^{r} Y \rightarrow J^{r} J^{r} Y$ depending on a sym-

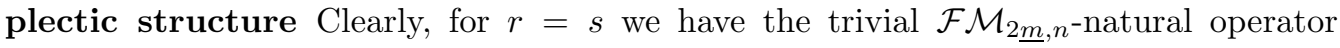
$A^{o}: \mathrm{SYMP} \rightsquigarrow\left(J^{r} J^{r}, J^{r} J^{r}\right)$ such that $A^{o}(\omega)=\mathrm{id}_{J^{r} J^{r} Y}$ for any $\mathcal{F} \bar{M}_{2 \underline{m}, n}$-object $Y \rightarrow M$ and any symplectic form $\omega$ on $M$. On the other hand, in the case $r=s$ we formulate the following hypothesis.

Hypothesis. There is no non-trivial $\mathcal{F} \mathcal{M}_{2 \underline{m}, n}$-natural operator $A$ : SYMP $\rightsquigarrow\left(J^{r} J^{r}, J^{r} J^{r}\right)$ transforming symplectic structures $\omega$ on $M$ into natural transformations $A(\omega): J^{r} J^{r} Y \rightarrow$ $J^{r} J^{r} Y$.

It seems that the verification of this hypothesis will be technically complicated. Bellow we prove only

Proposition 4. The hypothesis is true for $r=s=1$.

Proof. Suppose that there exists $A$ in question. Let

$$
\tilde{A}: J_{0}^{1} J^{1} \mathbf{R}^{2 \underline{m}, n} \rightarrow J_{0}^{1} J^{1} \mathbf{R}^{2 \underline{m}, n}
$$

be the restriction of $A\left(\omega^{o}\right)$ to the fiber over $0 \in \mathbf{R}^{2} \underline{m}$, where $\omega^{o}=\sum_{i=1}^{\underline{m}} d x^{i} \wedge d x^{\underline{m}}+i$ is the standard symplectic structure on $\mathbf{R}^{2} \underline{m}$. Quite similarly to the proof of Proposition 3 , 
$\tilde{A}$ is linear and we can write

$$
\tilde{A}\left(j_{0}^{1} j^{1}\left(x^{1}, 0, \ldots, 0\right)\right)=j_{0}^{1} j^{1}\left(a x^{1}+b \underline{x}^{1}+*, 0, \ldots, 0\right),
$$

where $^{*}$ is some linear combination of $x^{i} \underline{x}^{j}$ for $i, j=1, \ldots, 2 \underline{m}$. Using the invariance of $\tilde{A}$ with respect to the $\mathcal{F} \mathcal{M}_{2 \underline{m}, n}$-maps $\left(t i d_{\underline{m}} \times \frac{1}{t} i d_{\underline{m}} \times i d_{\mathbf{R}^{n}}\right)$ preserving $\omega^{o}$ we deduce that $*=0$. Analogously to the proof of Proposition 3 we deduce

$$
j_{0}^{1} j^{1}\left(b\left(\underline{x}^{1}\right)^{2}\right)=0,
$$

which reads $b=0$. Further, using the invariance of $\tilde{A}$ with respect to the $\mathcal{F} \mathcal{M}_{2 \underline{m}, n}$-map

$$
\left(x^{1}, \ldots, x^{2} \underline{m}, y^{1}-x^{1}, y^{2}, \ldots, y^{n}\right)
$$

preserving $\omega^{o}$ we get

$$
\tilde{A}\left(j^{1} j^{1}\left(x^{1}-\underline{x}^{1}, 0, \ldots, 0\right)\right)=j_{0}^{1} j^{1}\left(a x^{1}-\underline{x}^{1}\right) .
$$

Taking into account the $\mathcal{F} \mathcal{M}_{2 \underline{m}, n}$-map

$$
\left(x^{1}, \ldots, x^{2 \underline{m}}, y^{1}+\left(y^{1}\right)^{2}, y^{2}, \ldots, y^{n}\right)
$$

preserving $\omega^{o}$ we obtain

$$
\tilde{A}\left(j_{0}^{1} j^{1}\left(\left(x^{1}-\underline{x}^{1}\right)^{2}, 0, \ldots, 0\right)\right)=j_{0}^{1} j^{1}\left(\left(a x^{1}-\underline{x}^{1}\right)^{2}, 0, \ldots, 0\right) .
$$

Then as $j_{0}^{1} j^{1}\left(\underline{x}^{1}-x^{1}\right)^{2}=0$ and $j_{0}^{1} j^{1}\left(x^{1}\right)^{2}=0$, we have

$$
0=\tilde{A}\left(j_{0}^{1} j^{1}(0, \ldots, 0)\right)=\tilde{A}\left(j\left(\left(x^{1}-\underline{x}^{1}\right)^{2}, 0, \ldots, 0\right)\right)
$$

and

$$
j_{0}^{1} j^{1}\left(a x^{1}-\underline{x}^{1}\right)^{2}=-2(a-1) j_{0}^{1} j^{1}\left(x^{1}\left(\underline{x}^{1}-x^{1}\right)\right) .
$$

This yields $a=1$, i.e.

$$
\tilde{A}\left(j_{0}^{1} j^{1}\left(x^{1}, 0, \ldots, 0\right)\right)=j_{0}^{1} j^{1}\left(x^{1}, 0, \ldots, 0\right) .
$$

Considering the invariance of $\tilde{A}$ with respect to the $\mathcal{F} \mathcal{M}_{2 \underline{m}, n}$-map

$$
\left(x^{1}+x^{\underline{m}+1}, x^{2}, \ldots, x^{2 \underline{m}}, y^{1}, \ldots, y^{n}\right)^{-1}
$$

preserving $\omega^{o}$ and $j_{0}^{1} j^{1} x^{1}$ we deduce from (11)

$$
\tilde{A}\left(j_{0}^{1} j^{1}\left(x^{\underline{m}+1}, 0, \ldots, 0\right)\right)=j_{0}^{1} j^{1}\left(x^{\underline{m}+1}, 0, \ldots, 0\right) .
$$

Next, applying the invariance of $\tilde{A}$ with respect to permutations of first $\underline{m}$ base coordinates and respective second $\underline{m}$ base coordinates (preserving $\omega^{o}$ ) we deduce from (11) and (12)

$$
\tilde{A}\left(j_{0}^{1} j^{1}\left(x^{i}, 0, \ldots, 0\right)\right)=j_{0}^{1} j^{1}\left(x^{i}, 0, \ldots, 0\right)
$$

for $i=1, \ldots, 2 \underline{m}$. Then the invariance of $\tilde{A}$ with respect to the $\mathcal{F} \mathcal{M}_{2 \underline{m}, n}$-map

preserving $\omega^{o}$ yields

$$
\left(x^{1}, \ldots, x^{2 \underline{m}}, y^{1}+x^{j} y^{1}, y^{2}, \ldots, y^{n}\right)
$$

$$
\tilde{A}\left(j_{0}^{1} j^{1}\left(\underline{x}^{j} x^{i}, 0, \ldots, 0\right)\right)=j_{0}^{1} j^{1}\left(\underline{x}^{j} x^{i}, 0, \ldots, 0\right)
$$

for $i, j=1, \ldots, 2 \underline{m}$. Taking into account the invariance of $\tilde{A}$ with respect to the $\mathcal{F} \mathcal{M}_{2 \underline{m}, n}$ maps

$$
\left(x^{1}, \ldots, x^{2 \underline{m}}, y^{1}-x^{\beta}, y^{2}, \ldots, y^{n}\right)
$$


preserving $\omega^{o}$, where $\beta$ are $2 \underline{m}$-tuples of non-negative integers, from the clear equality $\tilde{A}\left(j_{0}^{1} j^{1}(0, \ldots, 0)\right)=j_{0}^{1} j^{1}(0, \ldots, 0)$ we get

and

$$
\tilde{A}\left(j_{0}^{1} j^{1}(1,0, \ldots, 0)\right)=j_{0}^{1} j^{1}(1,0, \ldots, 0)
$$

$$
\tilde{A}\left(j_{0}^{1} j^{1}\left(\underline{x}^{i}, 0, \ldots, 0\right)\right)=j_{0}^{1} j\left(\underline{x}^{i}, 0, \ldots, 0\right)
$$

for $i=1, \ldots, 2 \underline{m}$. Finally, using the linearity of $\tilde{A}$ and the invariance of $\tilde{A}$ with respect to permutations of fiber coordinates from (14),(15) and (16) we obtain $\tilde{A}=$ id (these elements generate the vector space $\left.J_{0}^{1} J^{1} \mathbf{R}^{2 \underline{m}, n}\right)$. Then $A$ is trivial because of the Darboux theorem, which is contradiction.

Denote by $\operatorname{ex}_{\Lambda}: J^{1} J^{1} \rightarrow J^{1} J^{1}$ the involution depending on a classical linear connection $\Lambda$ on the base of $Y$, which was constructed by M. Modugno [9]. Clearly, ex $\Lambda$ is a non-identic natural equivalence. From Proposition 4 it follows directly the following result, which was also proved in [8].

Proposition 5. Symplectic structures do not induce canonically classical linear connections.

REMARK 1. Let $\Gamma: Y \rightarrow J^{1} Y$ be a connection on a fibered manifold $Y \rightarrow M$. Using the exchange transformation $\operatorname{ex}_{\Lambda}: J^{1} J^{1} \rightarrow J^{1} J^{1}$, one can construct a connection $\mathcal{J}^{1}(\Gamma, \Lambda)$ on $J^{1} Y \rightarrow M$ by means of a classical linear connection $\Lambda$ on $M$ by

$$
\mathcal{J}^{1}(\Gamma, \Lambda):=\left(\operatorname{ex}_{\Lambda}\right)_{Y} \circ J^{1} \Gamma
$$

see [9]. By propositions 1, 2 and 4, it is impossible to construct in a similar way a connection on $J^{r} Y \rightarrow M$ from a connection $\Gamma$ by means of a symplectic form on $M$.

\section{References}

[1] M. Doupovec and I. Kolář, Iteration of fiber product preserving bundle functors, Monatshefte Math. 134 (2001), 39-50.

[2] M. Doupovec and W. M. Mikulski, On the involution of iterated bundle functors, Colloq. Math. 106 (2006), 135-145.

[3] C. Ehresmann, Extension du calcul des jets aux jets non holonomes, CRAS Paris 239 (1954), 1762-1764.

[4] I. Kolář, A general point of view to nonholonomic jet bundles, Cahiers Topol. Geom. Diff. 44 (2003), 149-160.

[5] I. Kolář, P. W. Michor and J. Slovák, Natural Operations in Differential Geometry, Springer-Verlag, 1993.

[6] I. Kolár̆ and M. Modugno, Natural maps on the iterated jet prolongation of a fibered manifold, Annali di Mat. Pura ed Applicata 157 (1991), 151-165.

[7] P. Libermann, Introduction to the theory of semi-holonomic jets, Arch. Math. (Brno) 33 (1977), 173-189.

[8] W. M. Mikulski, The natural bundles admitting natural lifting of linear connections, Demonstratio Math. 39 (2006), 223-232.

[9] M. Modugno, Jet involution and prolongation of connections, Čas. Pěst. Mat. 114 (1989), $356-365$. 